\title{
Testing odorant-receptor interaction theories in humans through discrimination of isotopomers
}

\author{
Mara Andrione, ${ }^{1}$ Marco Paoli, ${ }^{1}$ Renzo Antolini, ${ }^{1,2}$ Albrecht Haase ${ }^{1,2}$ \\ ${ }^{1}$ Center for Mind/Brain Sciences, University of Trento, Rovereto; ${ }^{2}$ Department of Physics, University of Trento, Povo, Italy
}

\begin{abstract}
Odour reception takes place on the olfactory receptor neuron membrane, where molecular receptors interact with volatile odorant molecules. This interaction is classically thought to rely on chemical and structural features of the odorant, e.g. size, shape, functional groups. However, this model does not allow formulating a correct prediction for the smell of an odorant, suggesting that other molecular properties may play a role in the odour transduction process. An alternative model of olfaction maintains that odorant receptors can probe not only the structural and chemical features, but also the molecular vibration spectrum of the odorants. This constitutes the so-called vibration model of olfaction. According to this model, two isotopomers of the same molecule,
\end{abstract}

Correspondence: Albrecht Haase, Laboratory for Nonlinear Bioimaging, Department of Physics \& Neurophysics Group, Center for Mind/Brain Sciences, University of Trento, Piazza Manifattura 1, Building 14, 38068 Rovereto (TN), Italy.

Tel.: +390464 808699

E-mail: albrecht.haase@unitn.it

Key words: olfaction, vibration model, isotopomer discrimination.

Authors' contributions: MA and MP designed the experiment, MA performed the experiment, MA and $\mathrm{AH}$ analysed the data, MA, AH, MP, RA discussed implications of the work, MA wrote the manuscript, $\mathrm{AH}$ and MP corrected the manuscript, $\mathrm{AH}$ acquired the funding.

Conflict of interest: the authors declare no potential conflict of interest.

Fundings: funding was provided by the Provincia autonoma di Trento (Research unit IBRAIM).

Acknowledgements: we thank Paolo Guerra for helping in the recruitment of participants and collection of data.

Received for publication: 15 June 2016.

Revision received: 6 January 2018.

Accepted for publication: 6 January 2018.

(C) Copyright M. Andrione et al., 2017

Licensee PAGEPress, Italy

Journal of Biological Research 2017; 90:6093

doi:10.4081/jbr.2017.6093

This article is distributed under the terms of the Creative Commons Attribution Noncommercial License (by-nc 4.0) which permits any noncommercial use, distribution, and reproduction in any medium, provided the original author(s) and source are credited. i.e. two forms of the same molecule, one unaltered and one in which one or more hydrogen atoms are substituted with deuterium - which are therefore structurally and chemically identical, but with different molecular vibration spectra - would interact differently with an olfactory receptor, producing different olfactory perceptions in the brain. Here, we report on a duo-trio discrimination experiment conducted on human subjects, testing isotopomer pairs that have recently been shown to be differentially encoded in the honeybee brain.

\section{Introduction}

Regarding the molecular basis of olfaction, there are still several unanswered questions, particularly concerning the interaction between odorant molecules and receptor proteins, which leads to olfactory signal transduction. ${ }^{1}$ Traditional views on the topic ${ }^{2}$ have been challenged by recent findings, ${ }^{3-5}$ but conclusive evidence could not be provided yet, leaving the debate widely open. ${ }^{6-9}$

Odour perception initiates on the membrane of olfactory receptor neurons, where volatile odorants interact with olfactory receptor proteins triggering a signalling cascade. As suggested by comparison with other receptors from the $\mathrm{G}$ protein-coupled receptor (GPCR) family, to which human odour receptors belong, ${ }^{1}$ such interaction might rely purely on chemical binding, probing the size, shape, and functional groups of an odorant. This model is predominant in textbooks, and constitutes the framework within which the majority of research on olfaction is conducted.

Although most of the scientific evidence on odour reception collected in different species is well in agreement with this model, ${ }^{10-13}$ it does not provide satisfactory explanation for the existence of many molecules of completely different chemical properties, i.e. size, shape, and functional groups, resulting in identical odours. ${ }^{14}$ At the same time, in large molecules, the substitution of a single inner atom, not contributing to chemical binding, can alter strongly the molecule's odour. ${ }^{15}$ Because of these inconsistencies, an alternative interaction mechanism has been proposed, connecting odour character to the vibration spectra of the odorants. ${ }^{16,17}$

Luca Turin suggested a first concrete physical explanation for vibration-sensing detection, which is based on an inelastic electron tunnelling spectroscopy mechanism. ${ }^{18}$ The theoretical feasibility of this ideas was supported by several quantum mechanical simulations. ${ }^{19-22}$ An experimental test of the vibrational model was suggested in Turin's original work: ${ }^{18}$ evaluating the odour discriminability of isotopomers, i.e. standard odorants and their deuterated counterparts, in which one or more hydrogen atoms were substituted for deuterium. These substitutions do not change chemical properties like shape, size, or functional groups of the molecules, but 
cause a substantial increase of the nuclear masses and therefore lead to major changes in the vibrational spectra. However, it has to be noted that some physical properties, such as the molecule's dispersion forces, are also slightly changed by the deuteration. ${ }^{23,24}$ By now, various behavioural studies of isotopomer discrimination were performed in humans, ${ }^{24-26}$ in Drosophila, ${ }^{20,27}$ and in honeybees. ${ }^{28}$ While outcomes of human tests were mixed, insect behavioural tests showed isotopomer discrimination. A first in vivo imaging experiment in the honeybee brain traced back the effect to the antennal lobe ${ }^{4}$ providing the first non-behavioural indication for different neuronal activation patterns corresponding to hydrogenated and deuterated odorants. In particular, response patterns to isotopomers of 1-octanol and benzaldehyde were clearly distinguishable. Recent electrophysiology experiments in Drosophila traced back differential responses to isotopomers to the odour receptor neurons of the antennae. ${ }^{3,5}$ However, a recent imaging experiment in Drosophila showed that an observed differential response to benzaldehyde in one olfactory receptor could be fully explained by a minute impurity of 6 ppm within the odour sample. ${ }^{29}$ Such impurity, undetectable by gas chromatography, had overshadowed the response to the nominal odorant.

Another study suggested limitations of theoretical models and reported a lack of isotopomer discriminations in an in vitro analysis of one human and several mouse olfactory receptors. ${ }^{7}$ However, the heterologous odour receptor expression in a kidney-derived cell line raised doubts about their functionality. ${ }^{1}$

Altogether, a final explanation of the molecule-receptor interaction is not in sight, and further experimental tests of any model will support this important research goal.

In the present study, we challenged human subjects with an olfactory discrimination task regarding the same pairs of isotopomers discriminated by honeybees. Overall results did not show a significant discrimination of hydrogenated from deuterated odorants, but an interesting trend in benzaldehyde suggests how to improve the design of future studies.

\section{Materials and Methods}

\section{Participants}

Subjects ( $n=201$, of which 108 females and 93 males) were recruited during a public event in Trento, Italy (La notte dei ricercatori) in September 2015. Their average age was 24.8 years (see Figure S6 for age distribution).

\section{Odorants}

Samples of the odorants were prepared a few hours in advance in $15 \mathrm{~mL}$ Falcon tubes. The odorants used were: 1-octanol (OCTh), purity $>99 \%$, and 1-octanol-d $\mathrm{d}_{17}\left(\mathrm{OCT}-\mathrm{d}_{17}\right), \mathrm{CD}_{3}\left(\mathrm{CD}_{2}\right)_{7} \mathrm{OH}$, purity $98.5 \%$, isotopic enrichment $98.8 \%$; benzaldehyde (BZA-h), purity $>99 \%$, and benzaldehyde-2,3,4,5,6- $\mathrm{d}_{5}\left(\mathrm{BZA}-\mathrm{d}_{5}\right), \mathrm{C}_{6} \mathrm{D}_{5} \mathrm{CHO}$, purity $99.9 \%$, isotopic enrichment $99.7 \%$. They were diluted 1:50 in mineral oil up to a $200 \mu \mathrm{L}$ volume. Those concentrations where chosen such that healthy subjects could clearly perceive the odour character of each compound. To exclude potential anosmic subjects, volunteers were asked whether they sensed a difference between OCT and BZA, which was answered positively by all participants. Data of those who could not provide clear answers to the tests were discarded.

\section{Behavioural task}

For the isotopomer discrimination tests, odorants were presented in three identical vials: one reference vial, and two test vials (Figure 1). One test vial contained the same isotopomer as in the reference vial, the other one a different isotopomer of the same odorant, forming a vial triplet for a so-called duo-trio test. In this duotrio discrimination task, a participant is asked to compare the reference with the two test vials and report which one of the two test vials had the same odour as the reference. There were two vial triplets prepared for benzaldehyde (one with BZA-h and one with BZA-d $\mathrm{d}_{5}$ as reference) and two for 1-octanol (one with OCT-h and one with OCT- $\mathrm{d}_{17}$ as reference). To perform a double-blind experiment, the four reference vials were marked with letters A-D and the test vials with numbers 1-8 by one experimenter. Each participant was randomly assigned by a second experimenter to two vial triplets, and then tested on both odorants, benzaldehyde and 1-octanol $(50 \%$ of participants, randomly assigned, in this order, and the other $50 \%$ in the reverse order). All experiments were conducted indoors within $3 \mathrm{~h}$ to guarantee stability of sample and ambient conditions.

\section{Statistics}

Data processing and statistical analyses were conducted respectively in $\mathrm{R}$ and Matlab. Percentages of correct answers were compared against chance level through one-tailed binomial tests; P-values throughout the text were Bonferroni-corrected for familywise errors (FWE).

\section{Results}

Responses to the tests were analysed for both isotopomer pairs: $1-$ octanol-h/d $\mathrm{d}_{17}$ and benzaldehyde-h/d $\mathrm{d}_{5} .98$ out of 198 subjects

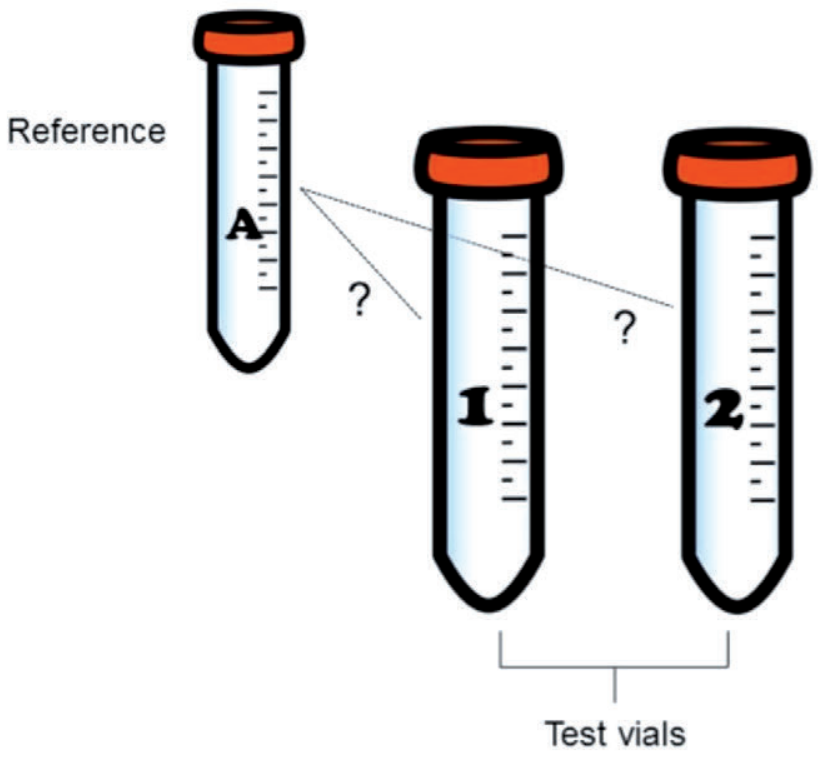

Figure 1. In the duo-trio test, participants are asked to first smell a reference vial and then confront it with two test vials. They are then asked to report which test vial contains the same odorant as the reference. 
could perform a correct distinction in the 1-octanol test, whereas 108 out of 198 correctly responded to the benzaldehyde test (Figure 2). Binomial one-tailed tests provided probabilities for deviation from chance level of $\mathrm{P}=0.94$ for 1 -octanol and $\mathrm{P}=0.23$ for benzaldehyde, respectively (Bonferroni corrected for the 2 tests). Although one might see a slight trend for discrimination of BZA, none of the two tasks was significantly solved.

To analyse the trend in BZA in more detail, we tested whether there was an effect of the order of presentation on the discrimination abilities of the subjects. From this analysis, a higher correct discrimination of the BZA isotopomers was found in those subjects performing first the BZA test: 63 out of 104 (Figure 3) with respect to those performing the BZA test after being tested on OCT: 45 out of 94 (Figure 3). A one-tailed binomial test on the subjects tested first on BZA provided a probability for deviation from chance of $\mathrm{P}=0.019$. However, the Bonferroni correction for FWE of the 4 potential tests on 2 odours and 2 administration orders reduces this value to a non-significant trend $(\mathrm{P}=0.078)$. In the case of 1 octanol, the presentation order was not affecting the proportion of correct answers (see Supplementary Figure S1).

Further exploratory analyses on potential effects of hydrogenated or deuterated odorants as reference (Supplementary Figures S2 and S3), ${ }^{25}$ of sex (Supplementary Figures S4 and S5), and of age (Supplementary Figures S6-S8) did not show significant deviation from chance level.

\section{Discussion and conclusions}

Discrimination of isotopomers has been suggested in various species, from insects ${ }^{20,27,28}$ to humans. ${ }^{24,25}$

Here, we tested the possibility that naïve human subjects may be able to discriminate two of the isotopomer pairs used in a previous study in honeybee: ${ }^{4} 1$-octanol and benzaldehyde. For both odorants, we performed duo-trio tests, revealing that the analysed overall population failed to significantly discriminate hydrogenated from deuterated odorants. This indicates that these isotopomer pairs could not be distinguished by human subjects under the tested conditions or, alternatively, that the number of discriminators in the tested population was too low to cause a significant effect. However, we noted that the order of odour presentation and/or the time between single tests should be carefully chosen in future studies, since those subjects that had first been tested on BZA showed a trend to correctly discriminate the isotopomers, while subjects tested on BZA after the OCT tests failed to deviate from chance level. This might indicate that when BZA was tested second, receptors activated by both odours might have still been saturated from the OCT test, rendering a discrimination of potential subtle differences between BZA isotopomers impossible.

Interestingly, the ability of human subjects to discriminate benzaldehyde isotopomers was previously tested, although with a dif-
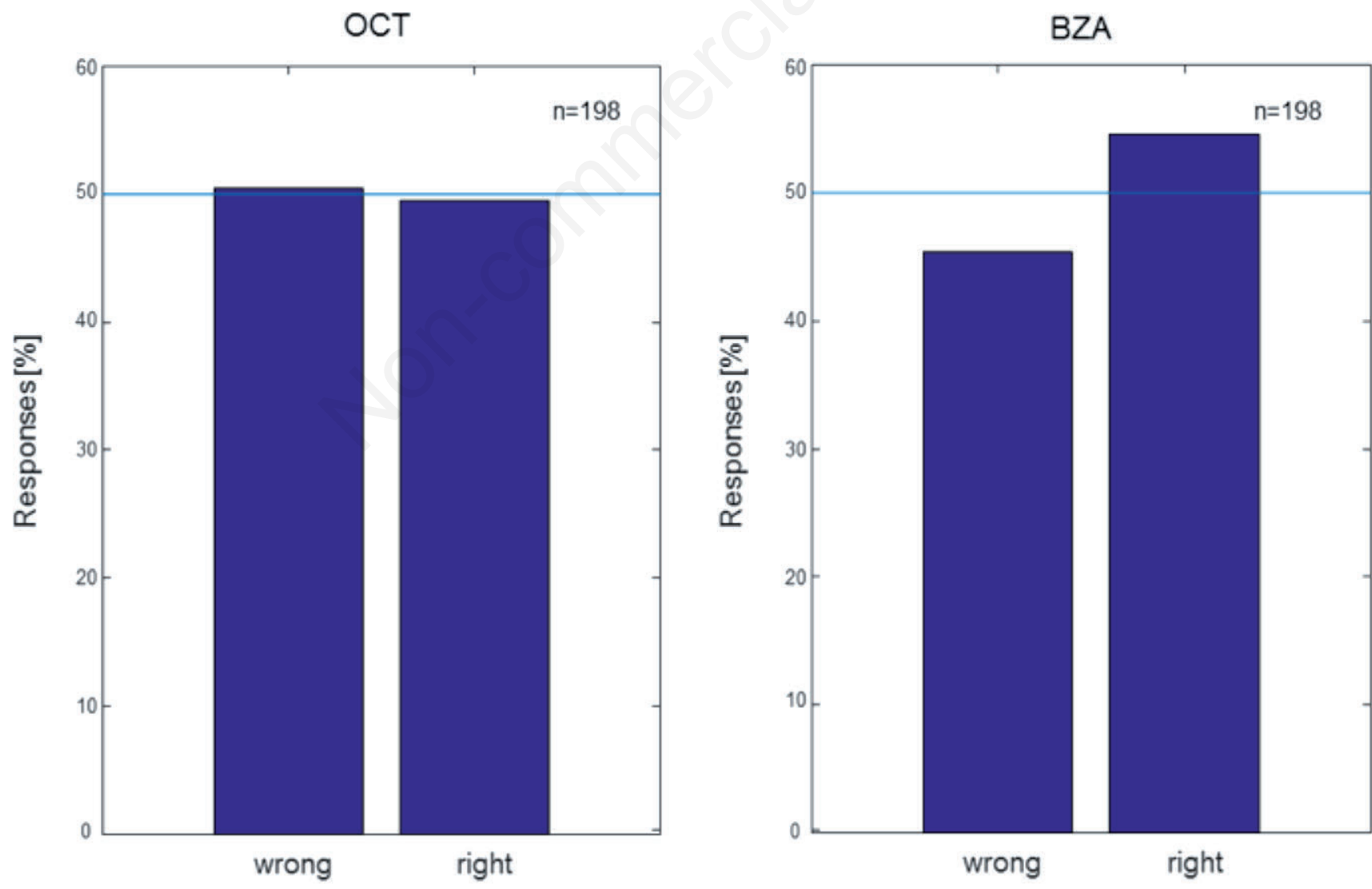

Figure 2. Percentage of wrong and right responses to the 1-octanol and benzaldehyde tests (absolute counts: wrong/right 100/98 and $90 / 108$, respectively). The line at $50 \%$ represents chance level. None of the two proportions of correct answers was significantly different from chance level (binomial one-tailed tests, respectively $P=0.95$ and $P=0.23$, Bonferroni corrected). 

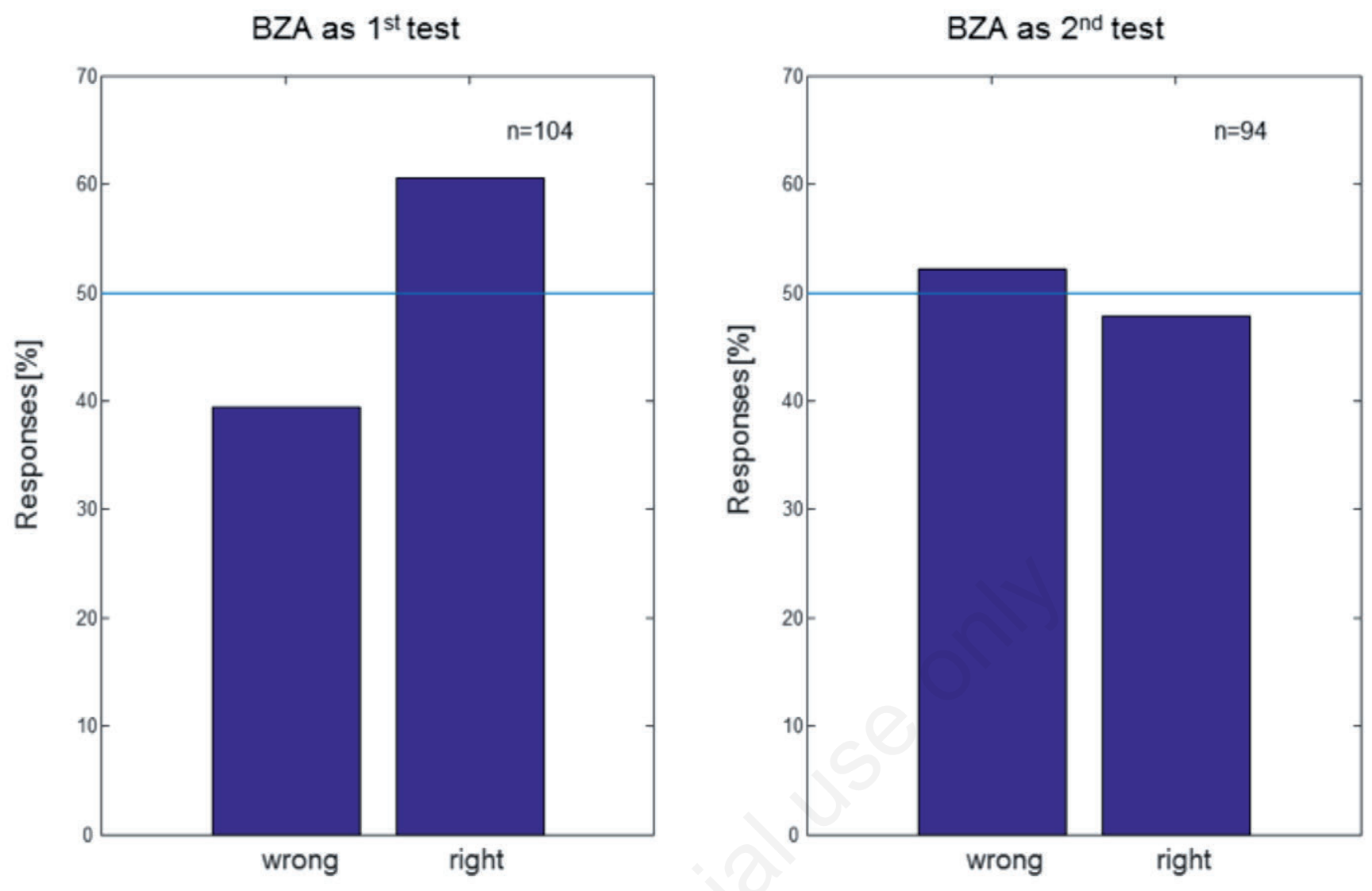

Figure 3. Percentage of wrong and right responses to the benzaldehyde (BZA) test, depending on the order of presentation. When the test on benzaldehyde was preceding that on 1-octanol (left plot, absolute counts: wrong/right $41 / 63$ ), there was a higher number of correct answers than in the benzaldehyde tests following 1-octanol tests (right plot, absolute counts: wrong/right 49/45). When all 4 odourorder combinations are tested, the FWE correction reduces the effect in the left plot below the significance level (binomial one-tailed test, $\mathbf{P}=\mathbf{0 . 0 7 8}$, Bonferroni corrected). The line at $50 \%$ represents chance level.

ferent protocol and a far smaller number of participants. ${ }^{25}$ In that study, the subjects were previously trained, to enhance their discrimination abilities, and a significant differentiation between BZA-h and BZA- $\mathrm{d}_{6}$ was reported. ${ }^{25}$ This discrepancy with our results might be explained by two factors. First, Haffenden et al. used a higher level of deuteration exchanging all 6 hydrogen atoms of the benzene ring, while we worked with the slightly lower deuterated $\mathrm{BZA}-\mathrm{d}_{5}$. The increase of isotopomer discrimination with the degree of deuteration was reported in several insect studies. ${ }^{4,20,27}$ On the other hand, the two studies looked at distinct aspects of olfactory discrimination. While the current report investigates the spontaneous discrimination abilities within a large population, Haffenden et al. tested this capability in a few trained subjects. This suggests that benzaldehyde isotopomers exhibit at most minor differences to human subjects, but training may enhance subjects' perception of these differences.

However, from our study we conclude that an influence of molecular vibrations on odour discrimination between the two tested isotopomer pairs could not be resolved in a broad naïve population. Our study further suggests that when several odours are tested, increased recovery times should be allowed between single tests, in order to avoid receptor saturation. In addition, we suggest to consider for future human studies also the results of a recently published insect experiment, ${ }^{29}$ showing that odour stimuli need to be purified by gas chromatography to avoid biases of impurities.

\section{References}

1. Horsfield AP, Haase A, Turin L. Molecular recognition in olfaction. Adv Phys X 2017;2:937-77.

2. Zarzo M. The sense of smell: molecular basis of odorant recognition. Biol Rev Camb Philos Soc 2007;82:455-79.

3. Drimyli E, Gaitanidis A, Maniati K, et al. Differential electrophysiological responses to odorant isotopologues in drosophilid antennae. eNeuro; 3. Epub ahead of print 21 June 2016. DOI: 10.1523/ENEURO.0152-15.2016.

4. Paoli M, Anesi A, Antolini R, et al. Differential odour coding of isotopomers in the honeybee brain. Sci Rep 2016;6:21893.

5. Maniati K, Haralambous KJ, Turin L, et al. Vibrational detection of odorant functional groups by Drosophila melanogaster. eNeuro 2017; [In press].

6. Vosshall LB. Laying a controversial smell theory to rest. Proc Natl Acad Sci U S A 2015;112:6525-6.

7. Block E, Jang S, Matsunami H, et al. Implausibility of the 
vibrational theory of olfaction. Proc Natl Acad Sci 2015;112: E2766-74.

8. Turin L, Gane S, Georganakis D, et al. Plausibility of the vibrational theory of olfaction. Proc Natl Acad Sci 2015;201508035.

9. Block E, Jang S, Matsunami H, et al. Reply to Turin et al.: Vibrational theory of olfaction is implausible. Proc Natl Acad Sci 2015;201508443.

10. Guerrieri F, Schubert M, Sandoz JC, et al. Perceptual and neural olfactory similarity in honeybees. PLoS Biol 2005;3:0718-32.

11. Bos N, d'Ettorre P, Guerrieri FJ. Chemical structure of odorants and perceptual similarity in ants. J Exp Biol 2013;216:3314-20.

12. Rizvanovic A, Amundin M, Laska M. Olfactory discrimination ability of Asian elephants (Elephas maximus) for structurally related odorants. Chem Senses 2013;38:107-18.

13. Yoder WM, Setlow B, Bizon JL, et al. Characterizing olfactory perceptual similarity using carbon chain discrimination in Fischer 344 rats. Chem Senses 2014;39:323-31.

14. Turin L. A method for the calculation of odor character from molecular structure. J Theor Biol 2002;216:367-85.

15. Sell CS. On the unpredictability of odor. Angew Chem Int Ed Engl 2006;45:6254-61.

16. Wright RH. Odour and molecular vibration. Nature 1966; 209:571-2.

17. Dyson MG. The scientific basis of odour. J Soc Chem Ind 1938;57:647-51.

18. Turin L. A spectroscopic mechanism for primary olfactory reception. Chem Senses 1996;21:773-91.

19. Brookes JC, Hartoutsiou F, Horsfield AP, et al. Could humans recognize odor by phonon assisted tunneling? Phys Rev Lett 2007;98:38101.

20. Bittner ER, Madalan A, Czader A, et al. Quantum origins of molecular recognition and olfaction in drosophila. J Chem Phys 2012;137:22A551.

21. Solov'yov IA, Chang P-Y, Schulten K. Vibrationally assisted electron transfer mechanism of olfaction: myth or reality? Phys Chem Chem Phys 2012;14:13861.

22. Reese A, Holmgaard List N, Kongsted J, et al. How far does a receptor influence vibrational properties of an odorant? 2016;1-21.

23. Turowski M, Yamakawa N, Meller J, et al. Deuterium isotope effects on hydrophobic interactions: the importance of dispersion interactions in the hydrophobic phase. J Am Chem Soc 2003;125:13836-49.

24. Gane S, Georganakis D, Maniati K, et al. Molecular vibrationsensing component in human olfaction. PLoS One 2013; 8:e55780

25. Haffenden LJW, Yaylayan VA, Fortin J. Investigation of vibrational theory of olfaction with variously labelled benzaldehydes. Food Chem 2001;73:67-72.

26. Keller A, Vosshall LB. A psychophysical test of the vibration theory of olfaction. Nat Neurosci 2004;7:337-8.

27. Franco MI, Turin L, Mershin A, et al. Molecular vibrationsensing component in Drosophila melanogaster olfaction. Proc Natl Acad Sci U S A 2011;108:3797-802.

28. Gronenberg W, Raikhelkar A, Abshire E, et al. Honeybees (Apis mellifera) learn to discriminate the smell of organic compounds from their respective deuterated isotopomers. Proc Biol Sci 2014;281:20133089.

29. Paoli M, Münch D, Haase A, et al. Minute impurities contribute significantly to olfactory receptor ligand studies: tales from testing the vibration theory. eNeuro 2017;4: ENEURO.0070-17.2017. 